\title{
Development of a Concept for Bridge Alert Management
}

\author{
F. Motz \& S. Hockel \\ Research Institute for Communication, Information Processing and Ergonomics, FGAN, \\ Wachtberg, Germany
}

M. Baldauf

World Maritime University, Malmö, Sweden

K. Benedict

Hochschule Wismar University of Technology, Business and Design, Warnemünde, Germany

\begin{abstract}
Modern ship bridges are highly-automated man-machine systems. Safe and efficient ship operations are dependent on the communication between humans and machines. This paper is dedicated to the general subject of integrated navigation and the specific field of the alert management on a ship's navigational bridge. It deals with investigations into the present situation on board of ships regarding the frequency and type of triggered alarms under real conditions. The conduction of empirical field studies is introduced and some of the gained results are presented and discussed. Finally the alert management concept of the performance standards for Integrated Navigation Systems (INS) is introduced and an approach for the reduction of CPA/TCPA alarm frequencies within INS/IBS is described.
\end{abstract}

\section{INTRODUCTION}

Modern ship bridges show a high degree of automation. A large amount of information concerning navigation, safety and security as well as the monitoring and control of the technical facilities on board are integrated in the operational displays on the bridge. With respect to the level of integration of the sensors, equipment, displays and assistance systems a modern ship bridge can be defined as a highlycomplex man-machine system. As such the safety and efficiency of its handling is dependent on the communication between the human and the machines during the accomplishment of tasks. Humans can fulfill their assigned monitoring, control, and decision tasks most effectively, if the information flow between them and machines is adapted to the human skills and abilities (e.g., Lützhöft 2004). To support the mariner effectively, information should be presented task- and situation-dependent.

Associated with the high degree of automation and integration of systems and sensors on board is a proliferation of alarm signals on the bridge. Redundant and superfluous audible and visual alarms are appearing, without a central position for visualiza- tion and acknowledgement of alarms. That way alarms increase the seafarers' workload and lead to information overload (Earthy 2006). Alarm signals coming from various systems and sensors lead sometimes to a confusing and difficult manageable situation for the mariner, which is distracting him from his task to safely navigate the vessel.

The majority of marine accidents are associated with collisions and groundings - in terms of numbers as well as in terms of costs. While some accidents result from technical failures of one kind or another (e.g. structural, engine or steering failure), many are caused by navigational errors (Wadsworth 2005). Research indicates that a high percentage of collisions and groundings are due to direct human error (IMO 2008).

To enable the operator to devote his full attention to the safe navigation of the ship and to immediately identify any abnormal situation requiring action to maintain the safe navigation of the ship an alarm management harmonizing the handling, distribution and presentation of alarms on the bridge is necessary (Bainbridge 1983, Sheridan 1998). 
For navigational alarms such an alarm management is introduced within the revised performance standards for integrated navigation systems (INS), which needs to be implemented for all INS installed after January $1^{\text {st }} 2011$. Additionally, the International Maritime Organization (IMO) decided to develop in the context of the revision of the performance standards for integrated bridge systems (IBS) a bridge alarm management system that comprises all alarms occurring on the bridge. A correspondence group coordinated by Germany was established to progress this work.

The importance of an alert management is recognized as well within the framework of the enavigation strategy of the IMO (IMO 2008). As one high-level user need within the e-navigation strategy an alarm management system as accomplished in the revised performance standards for INS is identified.

To investigate the current situation of the management and presentation of all alarms appearing on ships' bridges a number of field studies were performed on board of vessels. The results of those studies were introduced in the work of the IMO correspondence group working on the development of performance standards for Bridge Alarm Management. Within this paper selected results of the investigations are presented as well as the conclusions drawn regarding the performance standards for Bridge Alarm Management which will be finalized at the $55^{\text {th }}$ session of the IMO Subcommittee Safety of Navigation (NAV) in 2009. An approach for the reduction of the number of alarms is introduced.

The field studies were performed under the framework of a national Research and Development project funded by the German Ministry of Transport, Building and Urban Affairs, and under the European MarNIS-project, funded by the European Commission, Department for Energy and Transport.

\section{FIELD STUDIES - METHOD AND SAMPLE}

Field studies were carried out on board of six vessels: two ferries operating in the Baltic Sea, three container vessels (with container capacities of 6200 TEU, 5500 TEU and 7500 TEU) and a cruise vessel operating in the Mediterranean Sea. The aim of the field studies was to investigate the current occurrence of alarms on a ships bridge and their handling by the bridge team. As the management and presentation of alarms is influenced by the type of ship, the year of construction, the installed equipment and grade of integration, the sea area, the training and education of the crew as well as by the safety standards of the shipping company (Baldauf \& Motz 2006), these factors were taken into account to obtain a profound database.
All alarm and warning messages occurring on the bridge were manually recorded. It was registered what kind of alarm occurred, when and where it was announced, how it was presented and how it was handled by the bridge team.

To compare the different bridges on each vessel the specific sensor systems in use for navigation (positioning, speed, track control, collision avoidance and so on) together with the configuration of the alarm thresholds were registered. Simple changes in the configuration and the settings of the alarm limits lead to an increase or decrease of the announced alarms.

A special focus was laid on the assumed dependencies of alarm frequencies from sea areas. For the purposes of the studies the navigational situations were defined based on collected experts' opinions as:

- "open sea" (no natural constraints / no artificial constraints),

- "coastal" (natural constraints / distance to coast less than $10 \mathrm{~nm}$ without harbor, pilotage, anchorage / artificial constraints - e.g. TSS with established traffic lanes and recommended routes) and

- "confined" (with three different special cases: harbor, pilotage and anchorage).

Additionally interviews by means of a structured guideline were carried out to gather opinions, suggestions and remarks of navigators on the occurrence and presentation of alarms on the bridge, the handling of alarms and related operational problems.

The investigated vessels were built or reconstructed within the time span from 2001 until 2007. The ships' navigational bridges were provided with equipment from different manufacturers that was integrated and combined on a medium or high integration level.

The field studies were conducted on different times of the year during voyages in the Baltic Sea, in the Western Mediterranean Sea, in the North Sea and in the English Channel. Usually good weather conditions were experienced with low winds and calm sea. The average time of observation was 19 hours, with a minimum of 11 hours and a maximum of 27 hours.

\section{FIELD STUDIES - RESULTS}

\subsection{Alarm thresholds and adjustment of alarm announcement}

In the framework of the field studies it was recorded which settings the bridge team used for the triggering of alarms and warnings at the installed navigational equipment. It was assumed that the navigators 
would adjust the alarm thresholds and limit values to the different navigational situations and sea areas.

However the contrary was observed. On three of the six vessels no adjustments at all were made to the alarm thresholds and limit values - neither according to sea areas nor visibility conditions. On the remaining ships a change of the thresholds was rarely the case as well. One of the navigators adjusted the limits for CPA/TCPA (Closest Point of Approach/Time to CPA) in confined conditions to 0.5 nautical miles $(\mathrm{nm})$ and 0 minutes $(\mathrm{min})$ to practically switch off the alarms.

In general the thresholds for CPA alarms were set to $0.5 \mathrm{~nm}$ on five of the vessels with a TCPA limit of $6,10,12$ or (twice) 15 minutes. On Ferry 2 the CPA/TCPA limits were set to $0.0 \mathrm{~nm} / 0 \mathrm{~min}$ throughout the voyage.

Adjustments to the different navigational situations and sea areas were observed regarding the alarm announcement. The audible signaling of radar alarms was switched off from time to time on almost all vessels. Especially in confined conditions during departure and arrival, where a lot of targets appear on the radar, alarms (e.g. collision avoidance and lost target alarms) were not acoustically announced. On Ferry 2 the audible alarm announcement was switched off on both radars throughout the voyage.

\subsection{Dependencies on sea area}

One hypothesis of the investigations was that the frequency of alarms is dependent on the sea area in which the vessel is sailing. Figure 1 shows the average frequency of alarms per hour for the three sea areas for each vessel investigated.

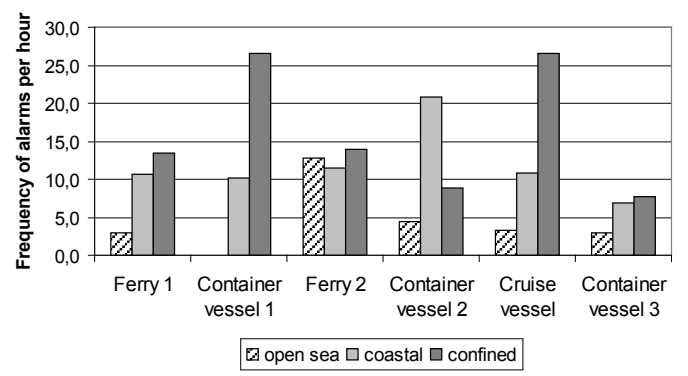

Figure 1. Average frequency of alarms for sea areas per vessel

Except for Ferry 2, on all vessels considerably more alarms were recorded in coastal and confined conditions than in open sea areas. Altogether the analyses indicate a correlation between the traffic characteristics of the specific sea areas and the alarm occurrence.

This hypothesis is further confirmed when analyzing the average frequency for all six vessels (Fig. 2 ). Most alarms were recorded in confined and coastal waters. The occurrence of alarms at open sea was approximately four times lower than in confined waters.

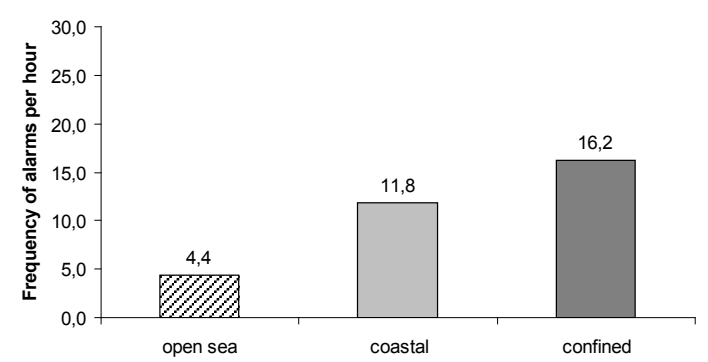

Figure 2. Average frequency of alarms for sea areas for all six vessels

\subsection{Dependencies on equipment}

Figure 3 depicts the distribution of alarms dependent on the device on which they occurred for the six vessels.

The vast majority of alarms was recorded at the radar device. Only on Container vessel 3 the percentage of radar alarms was lower than the amount of alarms that occurred at the ECDIS. This is due to the fact that on this vessel throughout the voyage AIS information was not integrated in the radar. Altogether the ECDIS aggregates the second highest percentage of the registered alarms.

The ECDIS was the only system besides the radar for which on each of the vessels alarms were recorded. Alarms on other devices were not appearing on all vessels, in some cases only on one vessel. These alarms reflected specific circumstances on the concerning vessel. On Container vessel 2 for example many alarms were registered on the GNSS and the gyro monitoring system, caused by the loss of the differential signal at the GNSS device.

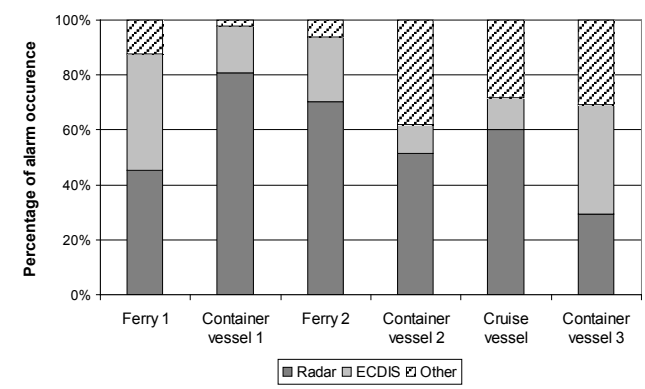

Figure 3: Distribution of alarms dependent on equipment per vessel

\subsection{Dependencies on alarm category}

The distribution of the five alarm categories registered most often is shown in Figure 4.

For the purpose of the analysis the percentages for off track and off course alarms were summed up into one category. The category "collision avoidance alarms" includes the values for CPA/TCPA and Bow 
Crossing alarms. Aggregated into the category "waypoint alarms" are the alarms: early course change indication, actual course change indication and wheel over point. Alarm types summed up under the category "other" partly took percentages of up to $17 \%$ on one vessel but were registered only on that vessel - as for example DGPS failures on Container vessel 2 .

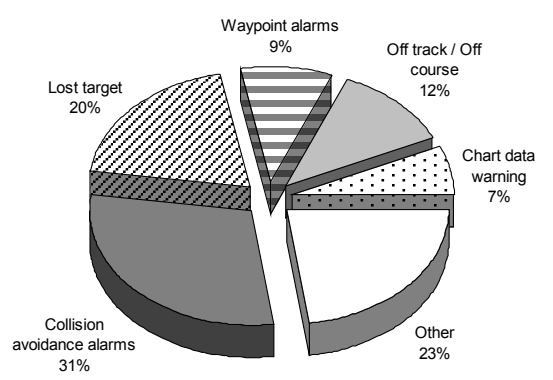

Figure 4: Average percentage of alarm categories for all six vessels

For all vessels investigated the majorities of alarms are collision avoidance alarms and lost target alarms (51\%).

CPA/TCPA as well as lost target alarms were predominantly triggered by AIS information, on average $72 \%$ of the CPA/TCPA alarms and $57 \%$ of the lost target alarms. (This percentage could have been even higher, if the bridge team of Container vessel 3 had not chosen a radar setting without integration of AIS information, which caused that all CPA/TCPA and lost target alarms were initiated by radar information.)

These results were expected due to the technical configuration and the use of the automatic alarm functions. For AIS, according to IMO regulations, the same limit values have to be applied as for tracked radar targets and the option for CPA/TCPA calculation was switched on to sleeping AIS targets by default. On two ships it was observed that CPA/TCPA and lost target alarms included targets that lay in the harbor basin behind land masses.

\subsection{Interview results}

During the field studies on board 13 mariners were interviewed. All of them were male with an average age of 36. Their average overall experience as mariner was 14 years, their average experience as an officer 9.5 years. The current position of the interviewees varied from master to third officer - most of the mariners were first or second officer.

Generally officers and masters feel that there are too many alarms occurring only for informative reasons on the bridge or can not be solved by the officer on the watch, for example "VDR record fault" or "window wiper oil low". Half of the mariners think that especially in certain situations for example the approach to a harbor such alarms are a major problem as they are distracting.

Mariners often reported alarms making annoying, loud and long lasting sounds, for example alarms from the navigation lights, echo sounder or gyro compass. Others pointed out that the acoustic presentation of an alarm often doesn't reflect its relevance. To identify the priority and the source of an alarm is seen as a general problem.

The majority favors to have the possibility to switch of the audible announcement of alarms in certain situations. Some mariners say that this concerns especially noisy alarms that are seen as less important. Others are referring to situations in which there is a lot of traffic, instruments are closely monitored and alarms are expected. However it is stated by some mariners that alarms for safety reasons, like fire alarms or engine shut down alarms, should always be audible.

Eighty percent think that a centralized alarm display for the centralized presentation of all alarms would support them with their tasks on the bridge. The vast majority feels that it would be a great benefit not to have to "run around the bridge" anymore trying to find out from which equipment an alarm comes from. Further expectations regarding a central alarm management display include a prioritization of alarms and the possibility to acknowledge or at least silence the alarms there. Some mariners worry that safety related alarms will not be immediately identified at a central alarm display for all alarms.

False alarms are seen as a problem. According to mariners especially distress alarms are often false alarms caused by users that send a message by mistake (without identification or position). Another problem are distress alarms from areas which are not of interest for the actual navigation situation.

Regarding the handling of alarms from the engine room presented e.g. on extension control panels on the bridge the statements differed. On some vessels the engine room is manned all or nearly all the time and alarms from the engine room are presented only for informative reasons without giving an audible announcement. On those vessels no difficulties with engine alarms are experienced. On other vessels alarms from the engine room give a sound on the bridge that stops, when the alarm is acknowledged in the engine room. On the bridge these alarms can not be acknowledged. It is only possible to silence the audible alarm. The tone is retriggered, when a new alarm occurs. This can lead to a distraction of the navigator, whose attention gets attracted by the sound again and again. Some mariners feel that those engine alarms are annoying. 


\section{DISCUSSION OF RESULTS}

The field studies indicate a lack of a harmonized alarm management. The majorities of alarms recorded were collision avoidance and lost target alarms occurring on the radar, being triggered by AIS information. The peak values of alarms per hour were observed in confined waters. Under conditions of high traffic density, as for example at harbor entrances, alarms were often experienced as superfluous. However alarm thresholds were rarely adjusted. Instead the audible alarm announcement on the radar often was switched off in confined conditions. On some vessels the bridge teams suppressed collision avoidance alarms during departure and arrival by setting the alarm thresholds to a minimum. Altogether it can be concluded that especially in confined waters as harbor approaches where many AIS targets appear the navigators are overloaded with alarms.

The results showed further, that difficulties are related to the audible presentation of alarms and the necessary acknowledgement on various panels on the bridge, to the lack of indication of any priority of the alarms, to the lack of a consistent alarm acknowledgment concept and to difficulties in differentiating the audible alarm signals.

\subsection{Generic Approach for Reduction of CPA/TCPA alarms}

As collision warnings were found a major part of all alarms on the bridge the situation regarding high number of alarms may possibly be improved by enhanced triggering of these type of alarm.

One reason for the high amount of collision avoidance and lost target alarms in confined waters is to be seen in the technical configuration of AIS and the integration of sleeping AIS targets for the presentation of collision avoidance and lost target alarms. According to the new display and the new radar standards (IMO 2004a, IMO 2004b), future radar systems with AIS integration will allow the selective acquisition of AIS targets for collision avoidance alarms and more flexibility for the presentation of lost target alarms. That way future radar system will have the capability to reduce the number of alarms. However, further studies are needed, to investigate if this really will solve the problem.

Further reasons for the high amount of alarms in confined waters are on the one hand the missing of recommendations for thresholds to be used for CPA and Bow Crossing limits taking into account relevant situation parameters and on the other hand the missing possibilities to adjust the alarm initiation in an appropriate way beyond range and time to con- fined waters, where closer passing of vessels are to be expected.

One concept for the improvement of collision warnings has already been described earlier by Baldauf (2004). It bases on the definition of situation dependent thresholds, which take into account the type of encounter situation, the sea area and the visibility conditions. Core element of this approach is a risk model for situation assessment defining the three types of encounters - meeting, overtaking and crossing courses - and considering the two conditions of good and restricted visibility as laid down in the International Rules for Preventing Collisions at Sea. To reduce the number of collision warnings the situation-dependent thresholds for CPA and TCPA can be applied by an algorithm for self-adaptation of these values to the prevailing circumstances of a certain situation and the maneuvering characteristics of the involved ships. According to first preliminary tests using a set of determined initial situationdependent CPA values to recorded open sea scenarios a significant reduction of occurring collision warnings was reached (Baldauf et al. 2008).

\section{PERFORMANCE STANDARDS FOR BRIDGE ALERT MANAGEMENT}

A lot of deficiencies observed in the field studies will be solved for INS installed after January $1^{\text {st }}$ 2011 for which an alarm management, according to the revised INS performance standards (IMO 2007), is mandatory.

This alarm management system for navigational alarms aims to harmonize the operation, handling, distribution and presentation of alarms. To improve the operator's situation awareness and his ability to take effective action a set of priorities is introduced based on urgency of the required response. A new philosophy is followed for the prioritization and categorization of alarms. Alert (alert management) is defined as umbrella term for the indication of any abnormal situation with three different priorities of alerts (IMO 2007, Motz \& Baldauf 2007):

- alarm (highest priority) - conditions requiring immediate attention and action by the bridge team to avoid any kind of hazardous situation and to maintain the safe navigation of the ship;

- warning - conditions or situations which require immediate attention for precautionary reasons, to make the bridge team aware of conditions which are not immediately hazardous, but may become so; and

- caution - awareness of condition which does not warrant an alarm or warning condition, but still requires attention out of the ordinary consideration of the situation or of given information. 
The three priorities are indicated visually and acoustically in different ways. Whereas alarms initiate an audible signal and a flashing visual indication until acknowledgement, warnings are presented with a momentarily audible signal and a flashing visual indication until acknowledgment. After acknowledgment both alarms and warnings are presented with a steady visual indication. Cautions are only indicated by a steady visual indication and don't have to be acknowledged. It is also possible to temporarily silence alarms.

To ensure a consistent presentation of alerts and to reduce the presentation of high priority alerts within the INS, alerts released by navigational functions, sensors, sources are presented as far as practicable, after evaluation with the system knowledge of the INS, e.g., provided by the integrity monitoring. This means that the priority of an alert will be assigned and presented consistently for all parts of the INS, and can be reduced for the alert in case of sufficient redundancy. E.g., in case of a failure of one of three position sensors only a caution may be released for the INS as still a reliable system position can be presented.

Additionally the INS performance standards include requirements for a central alert management human machine interface (HMI) for navigation related alerts (IMO 2007). Such a centralized presentation is part of an INS to support the bridge team in the immediate identification of any abnormal situation, including the source and reason for the abnormal situation and information for decision support for the necessary actions.

The central alert management HMI has to fulfill three major functions: indicating and identifying alerts, allowing to temporarily silence all alarms and allowing the acknowledgement of all alarms and warnings for which no additional graphical information is necessary as decision support for the evaluation of the alert related condition.

The alert management system within INS was developed with the intention to be extendable to an alert management concept for the whole bridge. The findings of the field studies showed that the aspects which were contributing to the development of the INS alert management are also to be applied to the alert management system for all alerts on the bridge.

Accordingly the performance standards for Bridge Alert Management, which is currently developed at IMO, picks up most of the ideas of the INS alert management. In doing so the performance standards consists of two major parts: A general module aiming to harmonize the presentation and handling of all alerts on the bridge (equivalent to the prioritization introduced within INS) and additionally requirements for a central alert management HMI.

\section{ACKNOWLEDGEMENTS}

The field studies were part of a project funded by the German Ministry of Transport, Building, and Urban Affairs, and conducted under the framework of the European MarNIS-project, funded by the European Commission, Department for Energy and Transport.

The authors would like to thank the shipping companies Peter Döhle, TT-Lines, Finnlines, Scandlines, HAPAG-Lloyd and AIDA Cruises Ltd for their grateful assistance and all mariners who provided their knowledge in interviews on board.

\section{REFERENCES}

Bainbridge, L. 1983. Ironies of Automation. Automatica 19: 775-779.

Baldauf, M. 2004. Enhanced Warning Functions for on board Collision Avoidance using AIS and VDR information. In R. Dauer, A. de la Pena, J. Puig (eds), International Congress on Maritime Technological Innovations and ResearchProceedings. Barcelona.

Baldauf, M.; Benedict, K. \& Motz, F. 2008. Aspects of Technical Reliability of Navigation Systems and Human Element in Case of Collision Avoidance. Proceedings of the Navigation Conference \& Exhibition, London, UK.

Baldauf, M. \& Motz, F. 2006. Operational Aspects of future Alert Management to support ship navigation. (in German) In Morten Grandt (ed.), Cognitive Systems Engineering in der Fahrzeug-undProzessfihnung. Bonn: DGLR-Bericht 2006-02 .

Earthy, J. 2006. Raising the alarm. Horizons 15: 10-11.

IMO 2004a. Performance standards for the presentation of navigation-related information on ship borne navigational displays. MSC.191(79). London: International Maritime Organization.

IMO 2004b. Revised performance standards for radar equipment. MSC.192(79). London: International Maritime Organization.

IMO 2007. Revised performance standards for integrated navigation systems (INS). MSC.252(83). London: International Maritime Organization.

IMO 2008. Report of the Subcommittee on Safety of Navigation to the Maritime Safety Committee. NAV 54/25. London: International Maritime Organization.

Lepsoe, A. \& Eide, M. 2005. Field Study on Bridge Alarms. Interim Sub-Task Report MarNIS WP2.4. Oslo: Det Norske Veritas.

Lützhöft, M. 2004. Maritime Technology and Human Integration on the Ship's bridge. Dissertation No. 907, Linköpping Studies in Science and Technology. Sweden.

Motz, F. \& Baldauf, M. 2007. Investigations into Shipborne Alarm Management - Conduction and Results of Field Studies. Proceedings of the 9th International Conference on Enterprise Information Systems, Volume HCI: 136-141. Funchal, Portugal.

Sherwood Jones, B.M.; Earthy, J.V.; Fort, E.; Gould, D. 2006. Improving the design and management of alarm systems. Proceedings of the World Maritime Technology Conference, London, March 2006.

Sheridan, T.B. 1998. Rumination on Automation. In S. Hishida; K. Inoue (ed.), Analysis, Design and Evaluation of Man-Machine-Systems. Reprints of the 7th IFAC/IFIP/IFORS/IEA Symposium, Kyoto.

Wadsworth, B. 2005. Marine eNavigation: An orientation paper. 9th world-wide electronic navigational chart database committee. WEND 9/INF.4. Monaco: International Hydrographic Organization 